\title{
FWEH induced high bootstrap current on Tore Supra
}

\author{
V. Basiuk, E. Joffrin, Th. Aniel, A. Bécoulet, G. T. Hoang, X. Litaudon, Th. Hutter, \\ F. Nguyen, B. Saoutic, W. A. Houlberg*, \\ C. E. Kessel \# \\ Association Euratom-CEA sur la fusion contrôlée, Centre d'Etudes de Cadarache \\ PEOEIVED \\ F-13108 Saint-Paul-Lez-Durance, France \\ * Oak ridge National Laboratory, Oak Ridge, Tennessee 37831 \\ \# PPPL, Princeton University, NJ 08543

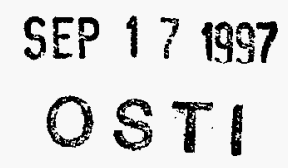

\begin{abstract}
Bootstrap current is regarded as a good candidate to sustain a large fraction of the plasma current, in the so-called "advanced" regimes of a tokamak reactor. It is thus important to study the stability of such discharges and to control them.

By means of fast wave electron heating (FWEH, up to $9.5 \mathrm{MW}$ ), stationnary high bootstrap discharges (during 5 secondes, $\AA 40 \%$ ) were routinely obtained on Tore Supra. The bootstrap profile is computed with a matrix formulation $(1,2)$ and is directly compared to the calculation of the non-inductive current.

The simulation of the loop voltage either with the code CRONOS (1D current diffusion code) using the profile of bootstrap current, or with the knowledge of the resistivity, allows also a self consistent determination of the bootstrap current. First results show that the energy enhancement factor $\mathrm{H}$ increases linearly with the fraction of bootstrap current.

The bootstrap induced by the FWEH is mainly due to the central pressure electron gradient (the central power deposition strongly peaks the electronic temperature). A OD study shows that the bootstrap current (Ibs) varies linearly with the poloidal beta $\quad\left(\mathrm{I} b s / p=C_{b s} \beta_{p}\right)$.

The effect of various plasma parameters (toroidal field $\mathrm{B}_{\mathfrak{t}}$, line-integrated density $\mathrm{n}_{\mathrm{l}}$, ion and electron temperature, plasma current $I_{p}$ ) on the bootstrap profile, fraction, $C_{b s}$ and on the confinement are analysed.
\end{abstract}

\section{BOOTSTRAP CURRENT INDUCED BY FWEH}

Fast Wave Electron Heating (dipole operation, up to $9.5 \mathrm{MW}$ ) induces high bootstrap discharges by means of an electronic central power deposition. The electronic temperature, strongly peaked, is responsible for more than $80 \%$ of the bootstrap current. The bootstrap profile is computed by two ways :

-a theoretical approach (with a matrix formulation $(1,2)$, valid in all collisionnality regime and aspect ratio) current.

-a determination of the non-inductive current which is, in our case, purely the bootstrap

A database of more than hundred shots (Fig. I) was obtained in various plasma conditions $\left(\mathrm{Te}(0)=2-8 \mathrm{keV}\right.$, ne $(0)=4.5-5.510^{19} \mathrm{~m}^{-3}, \mathrm{Ti}(0)=1-3 \mathrm{keV}, \mathrm{Ip}=0.4-1 \mathrm{MA}, \mathrm{B}_{\mathrm{T}}=2.2,2.8$ and 3.4 $\mathrm{T}$, Helium). Many shots are considered as stationnary shots (1 to 5 seconds) with respect to the diffusion time of the current. The electrons are in banana collisionnality regime, and the ions in the plateau regime. This database allows a study of the effect of plasma parameters on the bootstrap current profile.

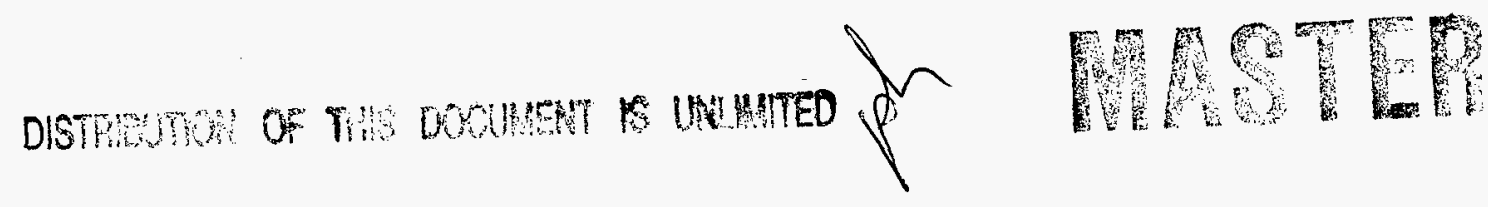




\section{DISCLAIMER}

This report was prepared as an account of work sponsored by an agency of the United States Government. Neither the United States Government nor any agency thereof, nor any of their employees, makes any warranty, express or implied, or assumes any legal liability or responsibility for the accuracy, completeness, or usefulness of any information, apparatus, product, or process disclosed, or represents that its use would not infringe privately owned rights. Reference herein to any specific commercial product, process, or service by trade name, trademark, manufacturer, or otherwise does not necessarily constitute or imply its endorsement, recommendation, or favoring by the United States Government or any agency thereof. The views and opinions of authors expressed herein do not necessarily state or reflect those of the United States Government or any agency thereof. 


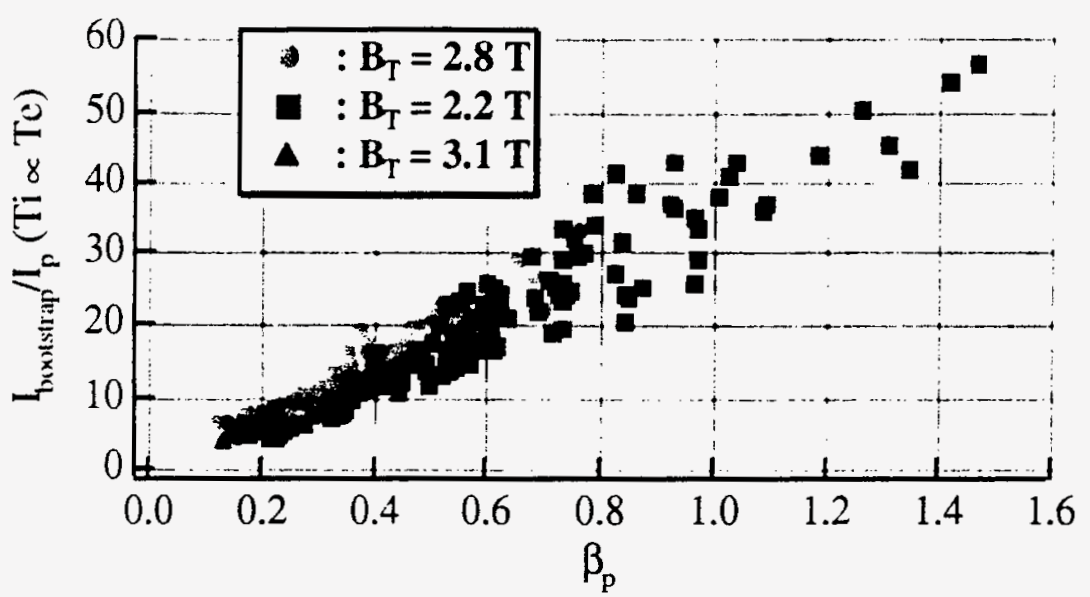

Figure I : Current bootstrap fraction versus poloidal beta

\section{Validation of the model}

The experimental bootstrap profile (non-inductive current) is determined by substracting the ohmic current profile from the total current. The ohmic fraction of the current is infered from the neoclassical resistivity and the lopp voltage profile calculated by time sequences of kinetic equilibria (3). This current is fairly compared to the theoretical estimation. The slight difference between the experimental and predicted profile (Fig. II b)) is well explained by the indetermination on the charge effective (Zeff).

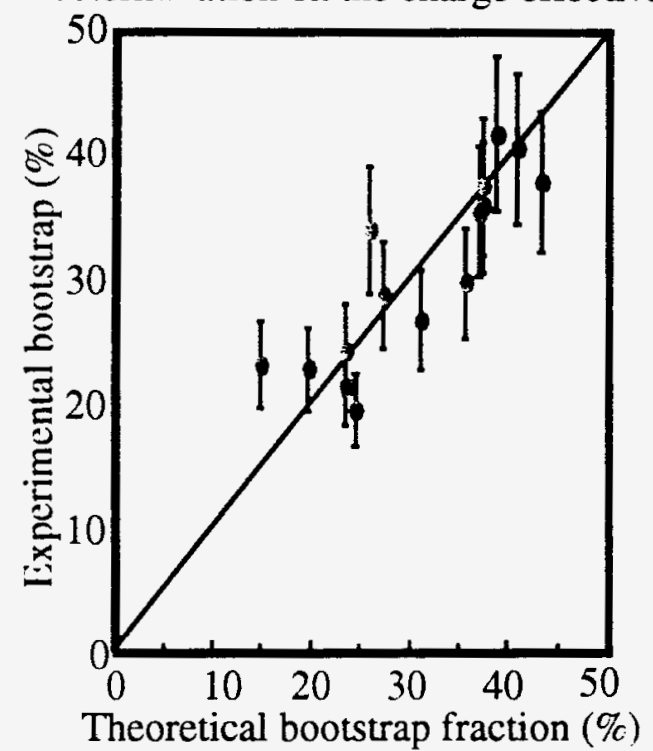

a)

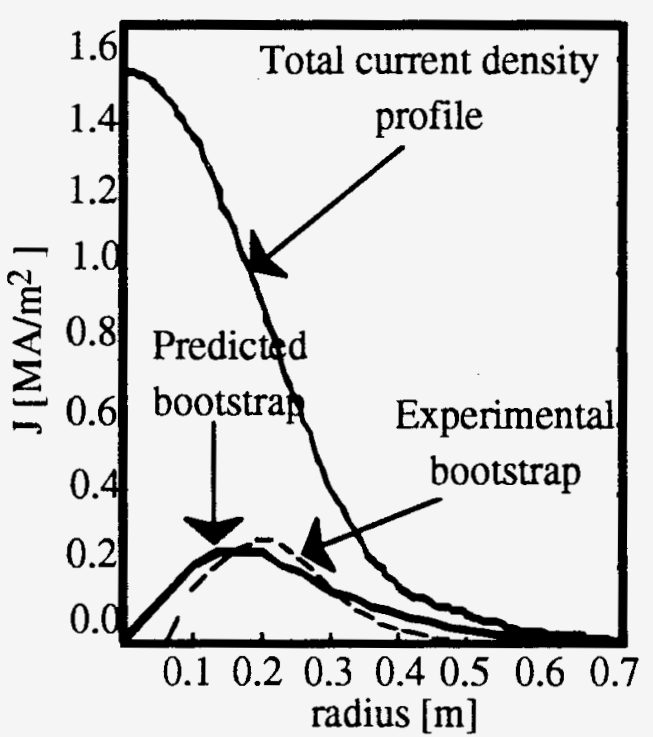

b)

Figure II : a) Comparison for twelve shots between the non-inductive current and the theoretical prediction of the current bootstrap fraction $\left.\left(B_{T}=2.2 T\right) b\right)$ Comparison of the profiles

Both determination of profiles requires the knowledge of Zeff. As the Zeff profile is not measured, it is assumed constant. But. recent measurements show that the Zeff profile is slightly peaked. which pushes the non-inductive current towards the center of the plasma whereas it has a small effect on the predicted profile. Then, the two profiles trend to match well.

Another strong assumption is made on the ionic temperature (Ti) profile. $\mathrm{Ti}$ is supposed to be either homothetic to the electronic temperature, or to the electronic density. The last 
hypothesis seems to be confirmed by new measurements of $\mathrm{Ti}$ made by charge exchange spectroscopy during ohmic discharges (4). The comparison between the two hypothesis (Fig. $3 a)$ ) allows an estimation of the error made on the bootstrap determination (Fig. 3b)) through the standard deviation of the gaussian fit : $\sigma \AA \AA A 10 \%$.
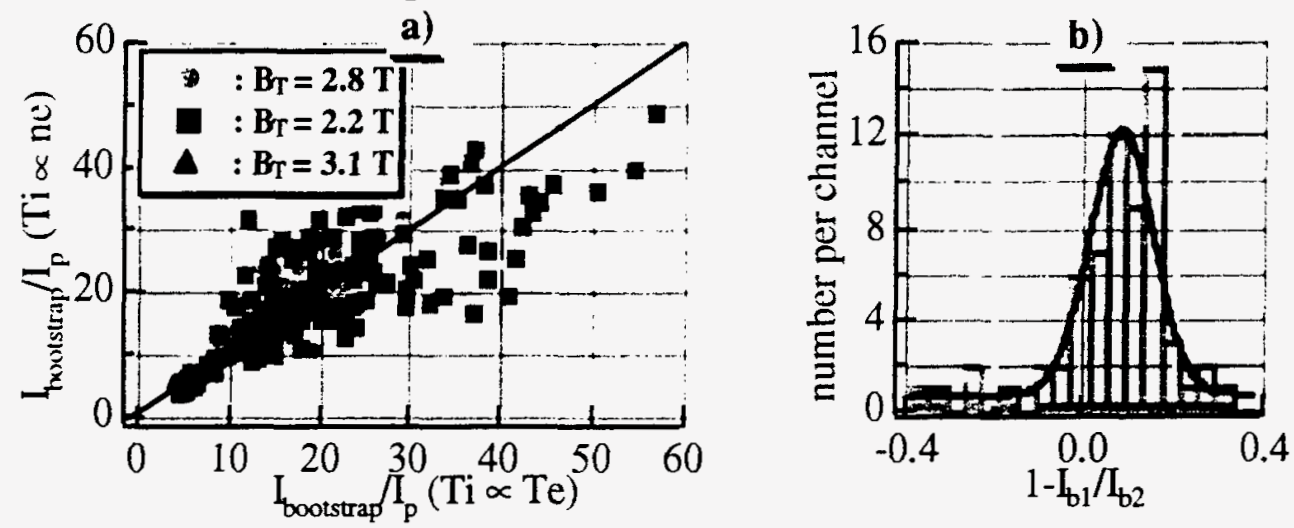

Figure III: a) Effect of Ti profile hypothesis (Ti propotionnal to ne or Te) on the current bootstrap fraction b) deviation between the two hypothesis $\left(1-b(T i \propto T e) / I_{b}(T i \propto n e)\right)$

The simulation of the loop voltage, by a 1D current diffusion code (5) using as input the predicted bootstrap profile strengthen the use of matrix formulation to calculate the bootstrap current (i.e. the Faraday rotation angles, q profile and li profile are well simulated).

\section{Scaling law for the bootstrap fraction}

A systematic study was made to find the plasma parameters which play a role in the bootstrap current. In the case of bootstrap induced by FWEH the main parameter is the poloidal beta $\left(\beta_{p}\right)$, as the fraction of bootstrap varies almost linearly with $\beta_{p}$ (Fig. I):

$$
\frac{I_{\text {bostrap }}}{I_{p}}=0.34 \beta_{p}^{1.09+0.04}
$$

No effect of temperature or density gradient is observed (one must note that for the density gradient, the range of variation is rather small).

$$
\begin{aligned}
& \frac{\mathrm{I}_{\text {ocostrep }}}{I_{\mathrm{p}}}=0.36 \beta_{\mathrm{p}}^{1.11 \pm 0.05} \mathrm{p}_{\mathrm{p}}^{-0.08+0.04} \mathrm{p}_{\mathrm{T}}^{0.03+0.08}, \\
& \mathrm{p}_{\mathrm{p}} \text { and } \mathrm{p}_{\mathrm{T}} \text { are the pression and temperature peakedness }
\end{aligned}
$$

The effect of $\operatorname{Te}(0)$ and $<\mathrm{n}>$, independently of $\beta \mathrm{p}$, can also be deduced from the database

$$
\frac{\mathrm{I}_{\text {boostrap }}}{\mathrm{I}_{\mathrm{p}}}=0.28 \mathrm{Te}(0)^{1.50 \pm 0.11}\langle\mathrm{n}\rangle^{0.43 * 0.19}
$$

The large error on the density coefficient is due to the small range of variation of $<n>$ $\left(<n>=3.3 \pm 0.810^{19} \mathrm{~m}^{-3}\right.$ for the database). The effect of temperature is clearly dominant over the effect of density.

\section{BOOTSTRAP DEPENDENCE ON TE AND NE PROFILE}

In the objective of increasing the bootstrap current fraction and of controling the bootstrap profile. it is essential to study the effect of Te and ne profile on the bootstrap current. 
The shape of the temperature (Te) and density (ne) profile can be defined with fourth parameters

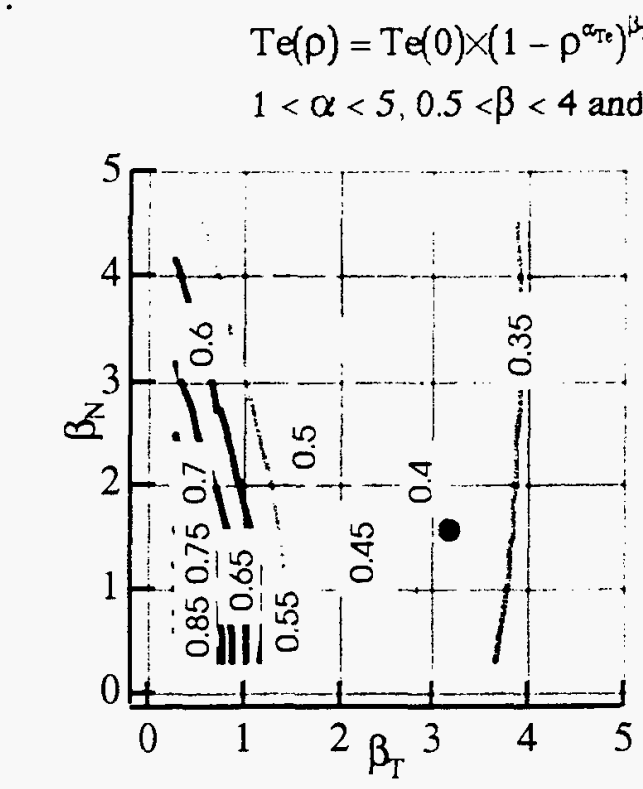

a)

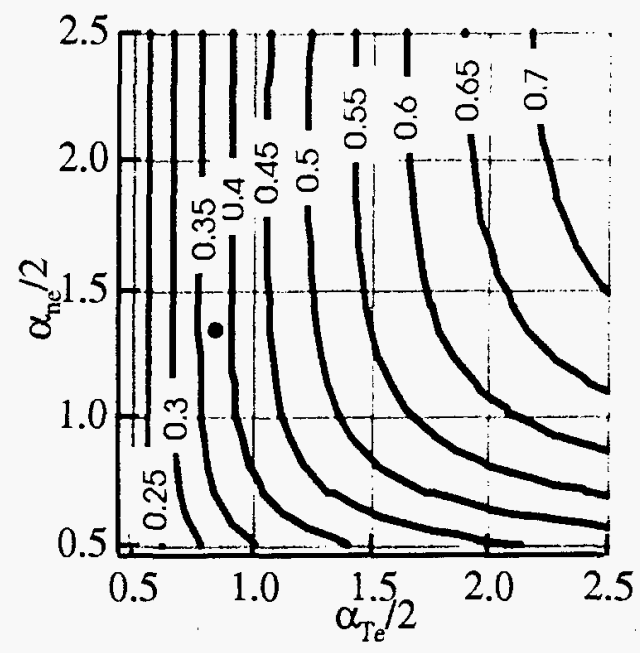

b)

Figure IV: Effect on the bootstrap fraction of a) peakedness profile ( $\beta$ ) b) gradient location $(\alpha)$ : shot 18805

The major trend of the simulation (Fig. 4) is that the temperature gradient has more effect on the bootstrap current than the density gradient. However, an increasing of the central density has the same effect on the current bootstrap than an increasing of the central temperature. Thus a pellet injection has an influence on the bootstrap fraction more by the fuelling effect than by a profile effect

Recent studies of non-inductive current during FWEH validated the use of matrix formulation to calculate the bootstrap current, show a major effect of the Te profile and leads to a TS scaling law for the bootstrap fraction.

\section{REFERENCES}

1. E. Joffrin et al, 22nd EPS Conference on controlled Fusion and Plasma Physics, Bournemouth 1995, Vol 19 C, part IV, p 125-128

2. C.E. Kessel, Nuclear Fusion. 34(9), (1994)

3. C. Forest et al, Physics Review Letters 73. p. 2444, 1994

4. Mandl. to be published at 24th EPS conference on controlled Fusion and Plasma Physics, Berchtesgaden, 1997

5. F. Kazarian-Vibert et al, $22^{\text {nd }}$ EPS Conference on controlled Fusion and Plasma Physics, Boumemouth 1995 , Vol 19 C, part III, p 373-376 
M97009357

(B) DOE, XF $($ B $U C-9,00,005$ DOE

19971202056 